SISSA-3/2003/EP

\title{
Aspects of String-Gas Cosmology at Finite Temperature
}

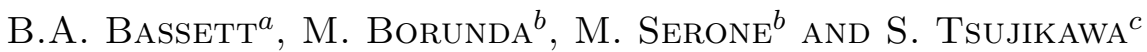 \\ ${ }^{a}$ Institute of Cosmology and Gravitation, University of Portsmouth, \\ Mercantile House, Portsmouth PO1 2EG, United Kingdom \\ ${ }^{b}$ ISAS-SISSA, Via Beirut 2-4, I-34013 Trieste, Italy \\ INFN, sez. di Trieste, Italy \\ ${ }^{c}$ Research Center for the Early Universe, University of Tokyo, \\ Hongo, Bunkyo-ku, Tokyo 113-0033, Japan
}

\begin{abstract}
We study string-gas cosmology in dilaton gravity, inspired by the fact that it naturally arises in a string theory context. Our main interest is the thermodynamical treatment of the stringgas and the resulting implications for the cosmology. Within an adiabatic approximation, thermodynamical equilibrium and a small, toroidal universe as initial conditions, we numerically solve the corresponding equations of motions in two different regimes describing the string-gas thermodynamics: (i) the Hagedorn regime, with a single scale factor, and (ii) an almost-radiation dominated regime, which includes the leading corrections due to the lightest Kaluza Klein and winding modes, with two scale factors. The scale factor in the Hagedorn regime exhibits very slow time evolution with nearly constant energy and negligible pressure. By contrast, in case (ii) we find interesting cosmological solutions where the large dimensions continue to expand and the small ones are kept undetectably small.
\end{abstract}




\section{Introduction}

String cosmology is rapidly growing in importance for at least two reasons. First, unless there is a fortunate conspiracy of scales and one or more of the extra dimensions are $\sim O\left(\mathrm{Tev}^{-1}\right)$ [1], we cannot reasonably expect to detect any stringy effects in accelerators in the foreseable future. Hence string cosmology may provide the only means of testing string or M-theory concretely, through e.g. non-commutative effects [2] or the AdS/CFT correspondence [3].

On the other hand, traditional cosmology has been fettered by the chains of the singularity theorems of General Relativity and is therefore usually only seen as understanding the evolution of our universe over the past 15 billion years or so since the Big Bang. String theory offers the exciting possibility of a resolution to the big bang singularity thereby opening up a potentially infinite pre-history of the big-bang. Models constructed in this vein include the pre-big-bang [4, 5] (see also 6, 7]) and the recent ekpyrotic/cyclic models [8, 9] which has lead to new work on string propagation in orbifold backgrounds with curvature singularities [10].

Traditional cosmology, because of the limitations of General Relativity, suffers from another great lacking: it is unable to make any predictions about the number of dimensions we live in or about the spatial topology of the Universe. String and M-theory theory, in contrast, predict that we live in either 10 or 11 spacetime dimensions. Perhaps the greatest challenge for string-cosmology, after understanding the big-bang singularity, is to explain why and how three dimensions became observable and large while 6 or 7 are either small or unobservable for some other reason. Perhaps the only proposal in this direction so far is the so-called BrandenbergerVafa (BV) scenario [11] where it is assumed that the universe is small and compact and that exactly three space dimensions become large because of the dynamics of winding modes, which play a particularly important role. Subsequently it has been pointed out in [4 that the low energy effective action of a dilaton-gravity system, naturally emerging in string theory, has a duality symmetry that is a manifestation of the string T-duality $R \rightarrow \alpha^{\prime} / R$ symmetry, that plays a crucial role in the analysis of [11. In this respect, the dilaton-gravity system is more suitable than standard General Relativity for the BV scenario [11]. Although some works have already shown that the BV scenario can be realized and extended in a variety of ways 12, 13. 14, 15, 16, 17, 18, 19, 20, 21, 22, (see also 23]), a full string-theory analysis is very complicated and still not completed, even in the simplest toroidal compactification.

In this paper, inspired by the BV scenario, we numerically solve the dilaton-gravity equations of motion [12] with some types of "stringy matter". Adiabatic evolution (which implies constant entropy), weak string coupling and thermodynamical equilibrium are always assumed in our analysis. For simplicity, we analyze Type IIA or IIB closed string theory on a $T^{9}$ torus, with no branes $^{1}$. In particular, we consider the following two regimes:

- (i) Hagedorn matter at high energy densities in a very small homogeneous and isotropic universe with a common compactification radius $\sim \sqrt{\alpha^{\prime}}$, and

- (ii) an almost-radiation dominated regime with two independent scale factors, associated with the large and small dimensions.

In the latter case, the lightest Kaluza Klein (KK) and winding mode contributions are also taken into account.

\footnotetext{
${ }^{1}$ In [17 it has been shown that fundamental strings, even in the presence of D-branes, are still the dominant degrees of freedom for the realization of the BV scenario.
} 
It is important to stress here that in both of these regimes, our matter is manifestly $T$ duality invariant. This symmetry is broken in our set-up only by the (arbitrary) choice of initial values. The main relevant questions in the two cases are respectively: which is the evolution of the universe at early times for a free Hagedorn string gas in thermal equilibrium? Assuming large and small dimensions as initial conditions, how do they evolve? In particular, do the small dimensions remain small?

String matter in the Hagedorn phase was already briefly discussed in [12] where it was realized that, to a first approximation in which the energy is constant, it leads to a very slow evolution of the universe, as in eq. (24). We complete the analysis of [12] by relaxing this approximation and imposing the conservation laws for KK and winding modes. We find that in any practical sense there is no departure from the behaviour dictated by eq. (24), and thus no relevant dynamics emerges in this set-up. It is important to recall how to interpret this result and its connection to the original BV scenario. The above result is obtained by assuming thermal equilibrium and a free, ideal, string gas, whereas the dynamics and interactions of winding modes at very early times are crucial in the BV scenario. The results we get in case (i) are therefore not closely connected with the original BV proposal [11] or, rather, they have to be taken the other way around. Namely, unless string interactions are taken into account and/or thermal equilibrium is relaxed, no interesting dynamics emerges.

On the other hand, the evolution of the small and large dimensions in case (ii) is much more interesting. First of all, we will show that when there is only pure radiation, the small dimensions can be stabilized and kept small relative to the large dimensions. Essentially, it is only required that the initial expansion rate of the large dimensions is bigger or of the same order than that of the small ones ${ }^{2}$. This mainly comes from the fact that the pressure in the small dimensions vanishes in the case of pure radiation.

When matter, in the form of KK and winding modes, is included, the choice of initial conditions becomes more relevant. The crucial point is played by winding modes that are able to distinguish large and small dimensions, leading respectively to a positive/negative contribution to the pressure along the large/small dimensions, as is clear from eqs. (46) and (47). This turns out in an expansion of the large dimensions and at the same time the possibility of keeping almost constant the small ones. In fact we found that there exists a wide range of parameters for which the small dimensions actually remain small (see fig. 4), while the large ones expand as required in the presence of radiation and string matter (see fig. 5). This is actually achieved for the natural initial condition where the small dimensions are close to the the self-dual radius $\sqrt{\alpha^{\prime}}$, together with the condition of the expansion rate mentioned in the pure radiation case.

These are the most important results of the paper. There exist a wide range of initial conditions for which the small dimensions are stabilized around the self-dual radius before entering into a purely radiation dominated phase, regime in which they are asymptotically stabilized anyway to a nearly constant value.

There are clearly several open issues that we do not consider but that deserve further investigation. First of all, thermal equilibrium, as well as isotropy and homogeneity along all directions, should be relaxed and string interactions need to be taken into account. Further, since toroidal compactifications are not realistic, always leading to non-chiral theories, study of more general topologies is required. See [19, 20] for recent progress in this direction.

\footnotetext{
${ }^{2}$ It is actually quite hard to imagine initial conditions where the expansion rate of the small dimensions are bigger than that of the large ones.
} 
Another very important issue unexplored in this work is the transition from dilaton gravity to General Relativity. A common lore is to assume that supersymmetry breaking occurs at later times and stabilizes all moduli, including the dilaton, which then becomes massive ${ }^{3}$. It is not clear how the evolution of the large and small dimensions are altered by this transition to an effective description in terms of General Relativity. Notice, however, that if one considers in case (ii) pure radiation with no matter, then the late-time behaviour of the dilatonic gravity solutions lead to a standard Friedmann-Robertson-Walker (FRW) radiation-dominated cosmology for the large dimensions, whereas the small ones are constant. This behaviour nicely matches with the solution found by [26] in General Relativity in a purely KK extra-dimensional scenario.

Finally, we assume an adiabatic approximation to simplify the analysis. A deeper justification of this approximation, or the dynamics which arises when it is dropped, is required. One can naively expect dropping this condition to yield a wider variety of dynamics, including more violent evolution.

This paper is organized as follows. In section 2 some general aspects of dilaton gravity are briefly reviewed. In section 3 we analyse the dynamics of the system in the extreme Hagedorn regime of high energy densities with a single scale factor. In section 4 the dilaton-gravity equations are solved in an almost-radiation dominated regime with large and small scale factors, as well as in the presence of some massive stringy matter. We present some conclusions in the final section and present some useful formulae in the appendix. We set the string scale $\alpha^{\prime}=1$ in the following.

\section{Dilaton gravity}

We shall study the dilaton gravity equations of motion with a massless dilaton field $\Phi$ corresponding to the low-energy effective action of string theory in $D+1$ space-time dimensions, described by [4, 5, 12,

$$
S=\int d^{D+1} x \sqrt{-g}\left[e^{-2 \Phi}\left\{R+4(\nabla \Phi)^{2}\right\}+\mathcal{L}_{M}\right],
$$

where $g$ is the determinant of the background metric $g_{\mu \nu}$, and $\mathcal{L}_{M}$ corresponds to the Lagrangian of some matter. The coupling of $\Phi$ with gravity is the standard one arising in string theory. Hereafter we shall consider the case with $D=9$. We are interested in the case where the whole universe is small and compact, corresponding to a flat $T^{9}$-torus. In this case, if one considers field configurations that are spatially homogeneous, assuming only slow time-dependence and adopting an adiabatic approximation it has been shown in [4, 12] that the action (11) exhibits a duality symmetry, a low energy manifestation of the string $T$-duality $R \rightarrow 1 / R$ symmetry. The ansatz for the metric and dilaton we use is

$$
d s^{2}=-d t^{2}+\sum_{i=1}^{9} R_{i}^{2}(t) d x_{i}^{2}, \quad R_{i}=e^{\lambda_{i}(t)}, \quad \Phi=\Phi(t) .
$$

Here the $R_{i}$ denotes the $i$-th scale-factor of the torus. The equations of motion simplify if one introduces a shifted dilaton, $\psi$, via

$$
\psi \equiv 2 \Phi-\sum_{i=1}^{9} \lambda_{i}
$$

\footnotetext{
${ }^{3}$ An alternative would be the models in which the dilaton stays massless but has universal couplings. It is even possible to reconcile this with quintessence if the dilaton has a divergent runaway behaviour [24] 25].
} 
Given the metric (2) the equations of motions of the dilaton-gravity system are then [12]

$$
\begin{aligned}
-\sum_{i=1}^{9} \dot{\lambda}_{i}^{2}+\dot{\psi}^{2} & =e^{\psi} E, \\
\ddot{\lambda}_{i}-\dot{\psi} \dot{\lambda}_{i} & =\frac{1}{2} e^{\psi} P_{i}, \\
\ddot{\psi}-\sum_{i=1}^{9} \dot{\lambda}_{i}^{2} & =\frac{1}{2} e^{\psi} E,
\end{aligned}
$$

with $E$ the total energy and $P_{i}$ the total pressure along the $i$-th direction found by multiplying the total spatial volume of the space by the energy density and pressure appearing in $\mathcal{L}_{M}$ of (11). Here a dot denotes derivative with respect to cosmic time, $t$. These equations are manifestly invariant under the duality symmetry [4, 12,

$$
\lambda_{i} \rightarrow-\lambda_{i}, \quad \Phi \rightarrow \Phi-\sum_{i} \lambda_{i}
$$

under which $\psi$ defined in eq. (3) is left invariant. It is typically assumed that the scale factors $R_{i}$ are the same in all directions, i.e. $R_{i}=R$. In contrast, we also consider in section 4 a scenario where the background is homogeneous and isotropic in $d$-spatial large dimensions and $(9-d)$ spatial small dimensions. We denote the large and small dimensions with their corresponding scale factors, as

$$
R=e^{\mu}, \quad r=e^{\nu} .
$$

In this case eqs. (4)-(6) take the form

$$
\begin{aligned}
-d \dot{\mu}^{2}-(9-d) \dot{\nu}^{2}+\dot{\psi}^{2} & =e^{\psi} E, \\
\ddot{\mu}-\dot{\psi} \dot{\mu} & =\frac{1}{2} e^{\psi} P_{d}, \\
\ddot{\nu}-\dot{\psi} \dot{\nu} & =\frac{1}{2} e^{\psi} P_{9-d}, \\
\ddot{\psi}-d \dot{\mu}^{2}-(9-d) \dot{\nu}^{2} & =\frac{1}{2} e^{\psi} E,
\end{aligned}
$$

where

$$
\begin{aligned}
& P_{d}=-\frac{\partial F}{\partial \mu_{i}}, \quad \forall i=1, \ldots, d, \\
& P_{9-d}=-\frac{\partial F}{\partial \nu_{i}}, \quad \forall i=d+1, \ldots, 9,
\end{aligned}
$$

in terms of the free energy $F$.

\section{Hagedorn regime}

According to the original BV proposal [11, the very early universe was compact over all nine spatial dimensions with radii $r \sim 1$ in string units. In this section we study the dilatongravity equations (4)-(6), with $E$ and $P$ the total energy and pressure of a free string gas in 
thermal equilibrium in such a compact, small universe. For simplicity, we consider the string gas associated with type IIA/IIB string theory compactified on a square $T^{9}$-torus, simple product of nine circles, with radii all equal to a common value $r=e^{\lambda}$. Although the strict thermodynamical limit $V \rightarrow \infty$ cannot be taken for this system, thermodynamics is still trustable as long as the system contains many degrees of freedom. In our case, this implies having an energy density $\rho \gg 1$. Our first step is then to derive the equation of state of the string matter in this regime, or equivalently the energy and pressure entering in eqs. (4)-(6).

\subsection{Microcanonical ensemble}

String thermodynamics in the high density phase has been a subject of intense study in the past (for a review see e.g. 27]). The most important point is the emergence of a critical temperature, the Hagedorn temperature $T_{H}$ [28], where the partition function of a free string gas diverges. It was soon realized that in this regime the usual thermodynamical equivalence between the canonical and microcanonical ensembles can break down and the latter, more fundamental ensemble, must hence be used. Furthermore, due to the presence of winding modes, the thermodynamical properties of a string system at finite volume differs substantially from that of an infinitely extended system [11, 29]. Following [29], it is useful to derive the energy density of states $\Omega(E)$ by analyzing the singularities of the one-loop string partition function in the complex $\beta$-plane, where $\beta=1 / T$ is the inverse temperature. This analysis has already been carried out in [29] but for completeness we report some useful details in the Appendix.

For a totally compact space at high energy, the leading singularity (a simple pole) of the partition function at $\beta=\beta_{H}$ is not sufficient to establish the thermodynamical properties of the system [11, 29. The first next-to-leading singularities are poles of order 18 with a dependence on the compactification radius. In this case a useful parametrization of the one-loop partition function $Z$ is given in eq. (57), from which one computes the density of states $\Omega(E)$ by means of eq.(58) and thus the associated entropy $S=\log \Omega(E)^{4}$. It reads

$$
S(E, r) \simeq \beta_{H} E+\log \left\{1-\frac{1}{\Gamma(18) \eta_{K W}^{18}}\left[\left(\eta_{K} E\right)^{17} \eta_{W}^{18} e^{-\eta_{K} E}+\left(\eta_{W} E\right)^{17} \eta_{K}^{18} e^{-\eta_{W} E}\right]\right\}
$$

where $\beta_{H}=2 \sqrt{2} \pi$, and

$$
\begin{aligned}
\eta_{K} & =\sqrt{2} \pi\left[2-\sqrt{4-\frac{2}{r^{2}}}\right], \\
\eta_{W} & =\sqrt{2} \pi\left[2-\sqrt{4-2 r^{2}}\right], \\
\eta_{K W} & =\sqrt{2} \pi\left\{\left[2-\sqrt{4-\frac{2}{r^{2}}}\right]-\left[2-\sqrt{4-2 r^{2}}\right]\right\} .
\end{aligned}
$$

The energy as a function of $r$ is given directly by (15), since $S=$ constant, by the assumption of adiabatic evolution. On the other hand, the temperature and pressure, defined as

$$
\frac{1}{T}=\frac{\partial S}{\partial E}, \quad P=\frac{T}{9} \frac{\partial S}{\partial(\log r)},
$$

\footnotetext{
${ }^{4}$ We have numerically checked that the term $\Lambda(\beta, R)$ in eq. (57) is negligible and thus is not reported in the following.
} 
yield

$$
\begin{aligned}
\frac{1}{T} & =\frac{1}{T_{H}}-\frac{1}{x E \Gamma(18)}\left[\left(\frac{z}{w}\right)^{18} y^{17}(17-y) e^{-y}+\left(\frac{y}{w}\right)^{18} z^{17}(17-z) e^{-z}\right], \\
P & =\frac{T}{9} \frac{\dot{x}}{x} \frac{1}{\dot{\lambda}}
\end{aligned}
$$

where

$$
x \equiv 1-\frac{1}{\Gamma(18)}\left(\frac{y z}{w}\right)^{18}\left(\frac{e^{-y}}{y}+\frac{e^{-z}}{z}\right),
$$

and $y \equiv \eta_{K} E, z \equiv \eta_{W} E, w \equiv \eta_{K W} E$. As usual in the microcanonical ensemble, the temperature is a derived quantity (from $S$ and $E$ ) and its explicit form is needed only to compute the pressure $P$. When the radius $r$ is close to unity, $T$ and $P$ are approximately given by

$$
\begin{aligned}
\frac{1}{T} & \sim \frac{1}{T_{H}}+C_{1} E^{17} e^{-\tilde{\eta} E}, \\
P & \sim C_{2} E^{17} e^{-\tilde{\eta} E},
\end{aligned}
$$

where $\tilde{\eta} \simeq \eta_{K} \simeq \eta_{W}$ for $r \sim 1$, and $C_{1}$ and $C_{2}$ are certain polynomial functions of $\tilde{\eta}$ and $\eta_{K W}$.

We numerically solved the dilaton-gravity equations (4)-(6) using a standard Runge-Kutta routine. We adopted initial conditions around $E_{0} \sim 1000, r_{0} \sim 1$, which comes from the requirement of T-duality. The shifted dilaton is chosen to satisfy the condition $e^{\Phi} \ll 1$ to ensure that the string coupling constant is initially small and hence that perturbation theory and the ideal gas approximation are trustable. The initial condition for $\dot{\lambda}$ is somewhat arbitrary and we have carried out simulations for a wide variety of different initial values of $\dot{\lambda}$. Notice that $\dot{\psi}_{0}^{2}$ is fixed by the constraint equation (44) and that the negative solution is taken to remain in the perturbative regime of small string coupling constant.

For initial conditions $r_{0} \in[0.8,1.2]$ and $E_{0} \sim 1000$, the temperature is very close to the Hagedorn temperature with a nearly constant value. This is clear from eq. (21), since the last term in (21) is vanishingly small relative to the first term, due to the exponential suppression given by $e^{-\tilde{\eta} E}$. Similarly we have $P \simeq 0$ for the above initial conditions from eq. (22). Therefore the system is effectively described by a pressureless dust as shown in fig. 1. In this case one has $\dot{\lambda} \simeq A e^{\psi}$ from eq. (5), with $A$ an integration constant. Subtracting eq. (6) from eq. (44), we find a simple relation, $\left(e^{-\psi} \psi\right)=E / 2$. Taking note that $E$ is nearly constant $\left(E \simeq E_{0}\right)$, the analytic solutions of eq. (4)-(6) in the Hagedorn regime may be written as

$$
\begin{aligned}
e^{-\psi} & \simeq \frac{E_{0}}{4} t^{2}+B t+\frac{B^{2}-d A^{2}}{E_{0}}, \\
\lambda & \simeq \lambda_{0}+\frac{1}{\sqrt{d}} \log \left|\frac{\left(E_{0} t+2 B-2 \sqrt{d} A\right)(B+\sqrt{d} A)}{\left(E_{0} t+2 B+2 \sqrt{d} A\right)(B-\sqrt{d} A)}\right| .
\end{aligned}
$$

$A$ and $B$ are integration constants depending on the initial values for $\dot{\lambda}, \psi$ and $\dot{\psi}$. In particular

$$
A=\dot{\lambda}_{0} e^{-\psi_{0}}, \quad B=-\dot{\psi}_{0} e^{-\psi_{0}},
$$

and $d$ is the number of dimensions (we are now considering the case with $d=D=9$ ). Notice that due to eq. (4), $\dot{\psi}_{0}$, and thus $B$, can not be taken to be vanishing. 


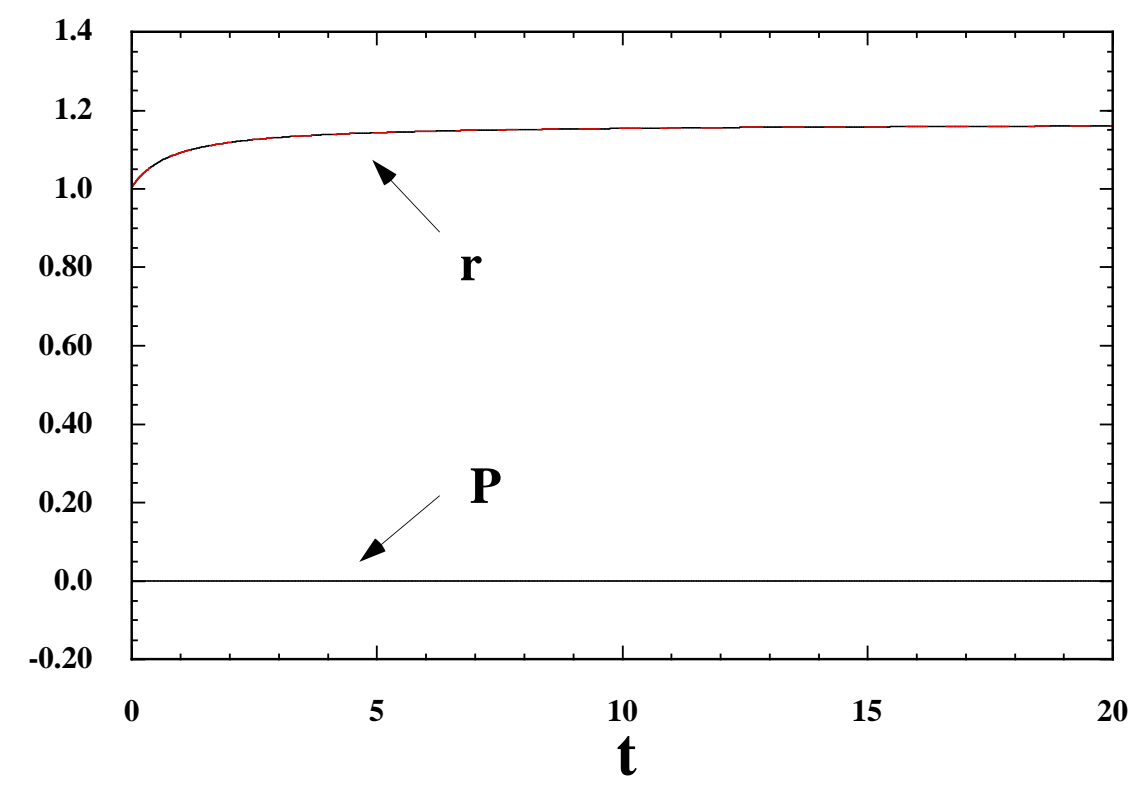

Figure 1: The evolution of $r$ and $P$ for the initial conditions $\lambda_{0}=10^{-3}, \dot{\lambda}_{0}=0.2, \psi_{0}=-5$ and $E_{0}=10^{3}$ in the Hagedorn regime. $\dot{\psi}_{0}$ is determined by the constraint equation (4). We plot the evolution of $r$ both using the analytic approximation (24) and the full numerical result, which show very good agreement each other.

In fig. 11we plot the evolution of $r$ that corresponds to the analytic solution (24), together with the full numerical results. They show very good agreement, which implies that the Hagedorn regime is well described by a state with a constant energy and negligible pressure. This actually ensures the validity of the analytic estimation in ref. [12] discussed briefly in its Appendix.

As long as $\dot{\lambda}_{0}$ is positive (negative), the radius grows (decreases) towards the asymptotic value

$$
r_{\infty}=e^{\lambda_{0}}\left|\frac{B+\sqrt{d} A}{B-\sqrt{d} A}\right|^{1 / \sqrt{d}},
$$

with $\dot{r}$ getting smaller with time (see fig. (1). We have checked this for values of $r_{0}$ very close to 1 , up to $r_{0}=\left(1+1 \times 10^{-15}\right)$, and found no substantial changes in behaviour. If one chooses exactly $r_{0}=1$, eq. (15) should be replaced by another similar relation, since now the two poles of order 18 in the $\beta$-plane approach each other to a single pole of order 36 . The above results apply also in this case: the pressure is almost zero and the evolution of the system is very slow in time.

For initial values of $E$ in the range $E_{0} \in[500,5000]$, the dynamics of the system is practically the same as explained above. For initial energies $E_{0} \gtrsim 1000$ the scale factor is essentially constant in time. On the other hand the system is typically unstable for $E_{0} \ll 1000$ and not thermodynamically meaningful for such low values of $E_{0}$. 


\subsection{Conservation laws}

We can also address the question of what happens when conservation laws are taken into account. In the case of the toroidal compactification we consider, the conserved quantities are taken to be the total winding number $N_{i}$ and the KK momenta $M_{i}$ in each compact dimension $(i=1, \ldots, 9)$. This is performed by introducing a chemical potential for each conserved charge 30.

A crucial difference with respect to the previous case is that the leading singularity of the partition function, namely the Hagedorn temperature, is enough to study the thermal behaviour of the system. In particular, the leading singularity now depends on the compactification radius induced by the conservation laws. The entropy of such an ideal gas can be easily computed, yielding

$$
S(E, r) \simeq \beta_{H} E-\frac{\pi}{4 \sqrt{2} E} \sum_{i=1}^{9}\left(\frac{M_{i}^{2}}{r^{2}}+N_{i}^{2} r^{2}\right)-9 \ln E .
$$

We see that in eq. (27) there are two suppression terms with respect to the leading term $\beta_{H} E$ in eq. (15). This is expected, because eq. (15) counts states with all values of charges whereas (27) counts a smaller set of states in which the value of the charge is fixed. Moreover, the number of states decreases as $M_{i}$ or $N_{i}$ are increased, since less energy is available for the oscillators. The temperature and pressure obtained from the entropy (27) are:

$$
\begin{aligned}
\frac{1}{T} & =\frac{1}{T_{H}}-\frac{9}{E}+\frac{1}{2 \sqrt{2} \pi} \frac{1}{E^{2}} \sum_{i=1}^{9}\left(\frac{M_{i}^{2}}{r^{2}}+N_{i}^{2} r^{2}\right), \\
P & =\frac{T}{9} \frac{\pi}{2 \sqrt{2}} \frac{1}{E} \sum_{i=1}^{9}\left(\frac{M_{i}^{2}}{r^{2}}-N_{i}^{2} r^{2}\right) .
\end{aligned}
$$

The pressure vanishes if one imposes vanishing winding and KK charge, $M_{i}=N_{i}=0$. The energy $E$ evolves very slowly for $E_{0}$ of order 1000 in which case the radius $r$ asymptotically approaches a constant value after some growth from $r_{0} \simeq 1$, thereby showing similar behaviour to fig. 1. When $M_{i}, N_{i} \neq 0$, due to presence of the $E$ factor in the denominator of eq. (29), the system evolves similarly to the case $M_{i}=N_{i}=0$, as long as the summation terms in eqs. (5) and (6) are unimportant. The dynamics changes if $M_{i}$ and $N_{i}$ are of order $10^{5}$, since the scale factor can have respectively a significant expansion or decreasing rate. As expected, winding modes prevent expansion, whereas KK modes, as standard matter, favorite it.

As a last remark, notice that the string gas defined by eqs. (28) and (29) has a negative specific heat. Systems with negative specific heat are unstable in non-compact spaces, but actually can be in thermal equilibrium in a finite space. Along the lines of 31, 32, we have evaluated the critical volume $V_{c}$ under which the system is in equilibrium and found that this is actually the case for $V_{c} \gg 1$, implying that the system can be actually in equilibrium with radiation. It should also be emphasized that this system is trustable as long as string corrections are completely neglected. When string interactions are included, the system is most likely to undergo a phase transition 33] whose details are so far unknown.

\section{Almost-radiation regime}

Assuming that some dimensions $(d)$ start to expand while the remaining $(9-d)$ dimensions remain small by means of some mechanism, the system will eventually reach a temperature 
below the Hagedorn regime where the dynamics is mostly governed by massless states, i.e. radiation $^{5}$. In this case, it is important to see the conditions under which the large dimensions continue to expand. At the same time, it is important to study whether the small dimensions remain small or if they also enter an expanding phase. Different from the Hagedorn regime discussed in the section 3, the microcanonical ensemble agrees with the canonical one for the range of temperatures and energy densities involved now. In the following we shall use the latter ensemble, which is more convenient for practical purposes.

Assuming again thermodynamical equilibrium and adiabatic evolution, we shall solve the dilaton-gravity equations (9)-(12), with $E$ and $P$ obtained from the free energy of a string gas at temperatures below $T_{H}$. The entropy $S$ is conserved with time under the assumption of adiabatic evolution:

$$
\frac{d}{d t} S=\frac{d}{d t}\left(\beta^{2} \frac{\partial F}{\partial \beta}\right)=0 .
$$

Eq. (30) is solved by letting $\beta$ and the scale factors $\lambda_{i}=\log R_{i}$, be slowly varying functions of time $\left[\beta \rightarrow \beta(t), \lambda_{i} \rightarrow \lambda_{i}(t)\right]$. In this way one can derive a differential equation whose solution gives $\beta=\beta\left(\lambda_{i}\right)$ with $S=$ constant. We denote the radii of the large $d$ dimensions, taken all equal, by $R=e^{\mu}$, whereas the radii of the $(9-d)$ small dimensions, again all equal, by $r=e^{\nu}$.

\subsection{Pure radiation}

As a first step, let us consider the case of pure radiation (see the Appendix for the free energy in the context of the canonical ensemble). The energy and pressure are easily evaluated from eq. (53) :

$$
E_{\text {rad }}^{(d)}=F_{\text {rad }}^{(d)}+\beta \frac{\partial F_{r a d}^{(d)}}{\partial \beta}=\frac{d R^{d}}{2 \pi} D(0)^{2} \Gamma\left(\frac{d+1}{2}\right)(4 \pi)^{\frac{d+1}{2}} \zeta(d+1)\left(1-2^{-(d+1)}\right) \beta^{-d-1},
$$

whereas the pressure $P_{\text {rad }}^{(d)}$ for the $d$ spatial dimensions is given by

$$
P_{\text {rad }}^{(d)}=-\frac{1}{d} \frac{\partial F_{r a d}^{(d)}}{\partial(\ln R)}=-F_{\text {rad }}^{(d)}=\frac{E_{r a d}^{(d)}}{d},
$$

which corresponds to the equation of state for radiation in $d$ spatial dimensions. Eq. (31) is nothing but the $d$-dimensional generalization of the Stefan-Boltzmann law in presence of $D(0)^{2} / 2$ bosonic and fermionic degrees of freedom. Since $F_{r a d}^{(d)}$ does not depend on $r$, the pressure along the small dimensions vanish:

$$
P_{\text {rad }}^{(9-d)}=-\frac{1}{9-d} \frac{\partial F_{r a d}^{(d)}}{\partial(\ln r)}=0
$$

From the adiabatic equation (30), we easily get the following relation

$$
\beta=\beta_{0} \frac{R}{R_{0}},
$$

\footnotetext{
${ }^{5}$ Notice that the system is already in an almost radiation regime for $\beta \gtrsim 11$ and it is essentially governed by pure radiation only for $\beta \gtrsim 14$, in string units.
} 
relating the temperature and scale factor in a radiation-dominated universe, with $\beta_{0}$ and $R_{0}$ being initial conditions satisfying $\beta\left(R_{0}\right)=\beta_{0}$. In this case the dilaton-gravity equations (9)(12) read

$$
\begin{aligned}
\ddot{\psi} & =\frac{1}{2} d \dot{\mu}^{2}+\frac{1}{2}(9-d) \dot{\nu}^{2}+\frac{1}{2} \dot{\psi}^{2}, \\
\ddot{\mu} & =\dot{\psi} \dot{\mu}+\frac{1}{2} e^{\psi} P_{\text {rad }}^{(d)}, \\
\ddot{\nu} & =\dot{\psi} \dot{\nu},
\end{aligned}
$$

together with the constraint equation

$$
\dot{\psi}^{2}=e^{\psi} E_{\text {rad }}^{(d)}+d \dot{\mu}^{2}+(9-d) \dot{\nu}^{2} .
$$

Eq. (37) is integrated to give

$$
\dot{\nu}=\dot{\nu}_{0} e^{\psi-\psi_{0}},
$$

where $\dot{\nu}_{0}$ are $\psi_{0}$ are the initial values of $\dot{\nu}$ are $\psi$. We see from eq. (39) that when $\dot{\nu}_{0}$ is positive (negative) the expansion rate for the small dimensions is always positive (negative). In order to avoid unbounded growth of the dilaton towards the strongly coupled regime $\left(e^{\Phi} \gtrsim 1\right)$, it is natural to consider the case with negative $\dot{\psi}$. In this case the absolute value of $\dot{\nu}$ decreases with time.

In the absence of the pressure $P_{\text {rad }}^{(d)}$ in eq. (36), the evolution of the large dimensions is similar to that of the small ones. In the case $\dot{\psi}_{0}<0$ and $\dot{\mu}_{0}>0$, we have $\ddot{\mu}<0$ for $P_{\text {rad }}^{(d)}=0$ from eq. (36). This corresponds to the universe with expanding large dimensions with a decreasing Hubble rate. Numerically we found that the evolution of the system in this case is trivial, namely the large dimensions soon approach a nearly constant value with very small $\dot{\mu}$.

When the pressure $P_{r a d}^{(d)}$ is taken into account, this works as a positive source term in eq. (36). Therefore it is possible to make the r.h.s. of eq. (36) positive even when $\dot{\psi}<0$ and $\dot{\mu}>0$. We have made numerical simulations with initial conditions $R_{0} \gg r_{0} \sim 1, \dot{\psi}_{0}<0$, and several different values of $\dot{\mu}_{0}$ and $\dot{\nu}_{0}$. As long as $\dot{\mu}_{0}$ is positive, the large dimensions expand in the presence of the pressure due to radiation. The contribution of the pressure term in eq. (36) inhibits the rapid decrease of $\dot{\mu}$, thereby leading to different evolution of $R$ compared to the case with $P_{\text {rad }}^{(d)}=0$. The expansion rate $\dot{\nu}$ for the small dimensions is exponentially suppressed with the decrease of $\psi$ [see eq. (39)] ]. Therefore unless the initial value of $|\dot{\nu}|$ is much larger than unity, the radius $r$ can stay small around $r \sim 1$.

We have numerically succeeded to obtain ideal solutions with growing $R$ and small, roughly constant, $r$ satisfying $r \ll R$. One typical evolution is plotted in fig. 2. These solutions can be achieved by choosing initial values with $\dot{\mu}_{0} \gtrsim\left|\dot{\nu}_{0}\right|, R_{0} \gg r_{0} \sim 1$ and $\dot{\psi}_{0}<0$. When $\dot{\mu}_{0} \ll\left|\dot{\nu}_{0}\right|$ holds initially, it is difficult to keep the small dimensions small relative to the large ones. If $\dot{\mu}_{0}<0$, we have $\ddot{\mu}_{0}>0$ from eq. (36). This leads to the growth of the expansion rate $\dot{\mu}$. Since $\dot{\mu}$ continues to be negative by the time it crosses zero, the large dimensions contract during this stage. After $\dot{\mu}$ changes sign, $R$ begins to grow. This implies that bouncing solutions may be obtained if $\dot{\mu}_{0}<0$. We have numerically found that this is actually the case, see fig. 3. During the contracting phase, the temperature increases according to eq. (34). The temperature is maximum at the bounce where $R$ is minimum. In the context of Pre-Big-Bang [5] or Ekpyrotic 


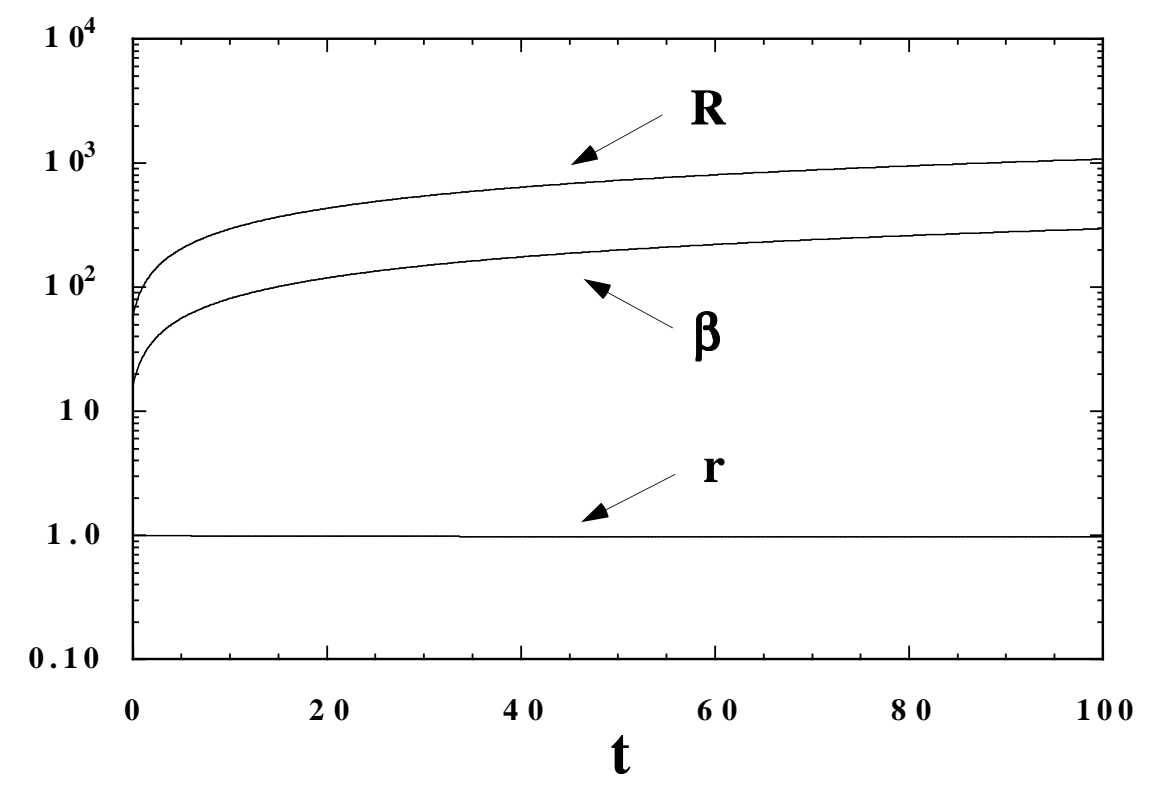

Figure 2: The evolution of $R, r$ and $\beta$ for the pure radiation case with $d=3$. We choose the initial conditions $\dot{\mu}_{0}=1.0, \mu_{0}=4.0, \dot{\nu}_{0}=-0.01, \nu_{0}=0.0, \psi_{0}=-16$ and $\beta_{0}=15$.

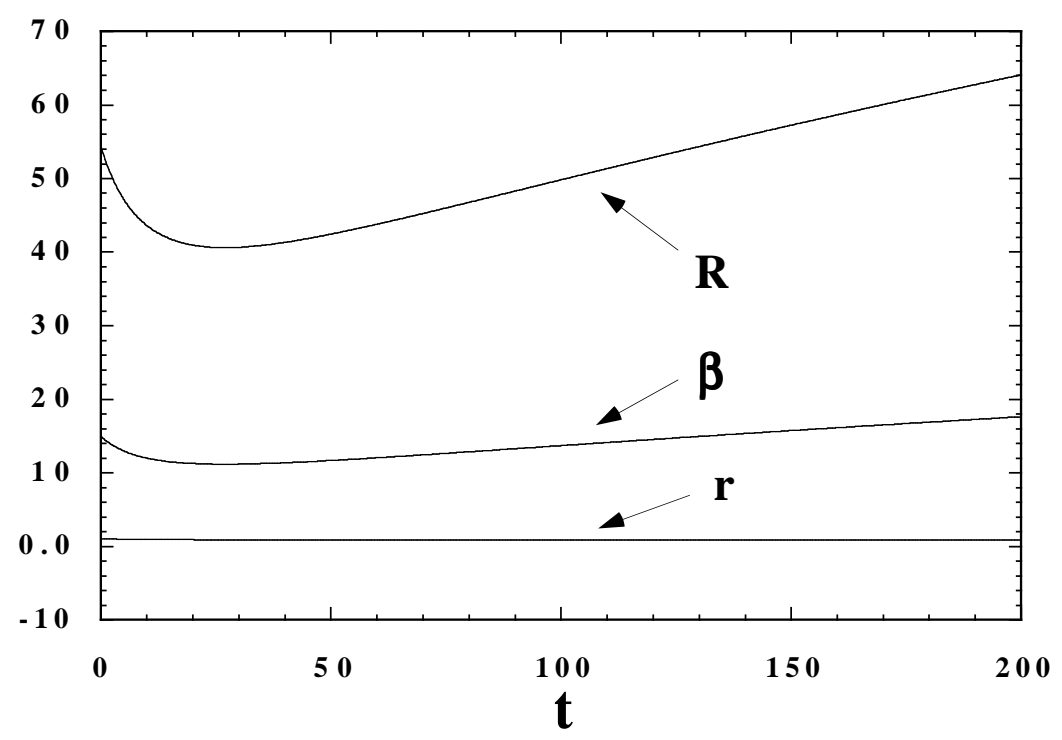

Figure 3: The evolution of $R, r$ and $\beta$ for the pure radiation case with $d=3$. We choose the initial conditions $\dot{\mu}_{0}=-0.04, \mu_{0}=4.0, \dot{\nu}_{0}=-0.01, \nu_{0}=0.0, \psi_{0}=-16$ and $\beta_{0}=15$. 
Cosmologies [8], nonsingular bouncing solutions are difficult to construct unless loop or derivative corrections are added to the tree-level action 34, 35. It is quite interesting to be able to obtain bouncing solutions only by including radiation in dilaton-gravity equations.

It is worth investigating the asymptotic behavior of the dynamical system of eqs. (35)-(37), along the lines of [12]. Introducing new parameters, $\dot{\mu} \equiv \xi, \dot{\nu} \equiv \eta, \dot{\psi} \equiv f$, and using the fact that the pressure in the large dimensions is connected to the energy $E_{\text {rad }}^{(d)}$ through eq. (32), eqs. (35)-(38) become:

$$
\begin{aligned}
\dot{f} & =\frac{d}{2}\left[\xi^{2}+\left(\frac{9}{d}-1\right) \eta^{2}\right]+\frac{1}{2} f^{2}, \\
\dot{\xi} & =-\frac{1}{2}\left[\xi^{2}+\left(\frac{9}{d}-1\right) \eta^{2}\right]+f \xi+\frac{f^{2}}{2 d}, \\
\dot{\eta} & =f \eta
\end{aligned}
$$

Since we are considering the case with decreasing $\dot{\psi}, \eta$ asymptotically approaches zero from eq. (39), i.e. $\eta=0$ is an attractive solution. In this case, the analysis is closely related with the one outlined in the Appendix of [12. In particular one finds that the line described by

$$
f / \xi=-d, \quad \eta=0,
$$

is an attractor. From eq. (3) the time-derivative of the dilaton is given as $2 \dot{\Phi}=f+d \xi+(9-d) \eta$. Therefore we have $\dot{\Phi}=0$ for the attractor (43), again in complete analogy to the case of the single scale factor [12]. Substituting eq. (43) for eq. (41) and integrating this relation, one finds

$$
\xi \propto \frac{2}{(d+1) t}, \quad R \propto t^{\frac{2}{d+1}} .
$$

This indicates that the late time evolution for the large dimensions can be described by that of the standard radiation dominant phase in FRW cosmology for $d=3$, even in the presence of the small dimensions. The key point is that the pressure in the small dimensions vanishes for the massless case, thereby leading to $\eta=0$ as an attractor. Notice that a cosmological solution of this kind has been also obtained in standard General Relativity and in a purely Kaluza-Klein extra-dimensional scenario by [26].

We also analyzed the evolution of the system by varying the value of $d$, and found that the situation is not basically changed compared to the $d=3$ case discussed above. As long as the initial conditions satisfy $\dot{\mu}_{0} \gtrsim\left|\dot{\nu}_{0}\right|, R_{0} \gg r_{0} \sim 1$ and $\dot{\psi}_{0}<0$, the large dimensions continue to expand due to the presence of radiation while the small dimensions are kept to be small $(r \ll R)$. In this case the large dimensions asymptotically approach the radiation-dominant FRW solution (44). When the initial value of $\dot{\mu}$ is negative, we found that it is possible to have a bouncing cosmological solution that approaches the expanding FRW universe given by (44).

\subsection{Inclusion of matter}

Although massless states, pure radiation, dominate the thermodynamical ensemble in this phase, this dominant contribution has a trivial dependence on the small dimensions $r$. In particular, $F_{\text {rad }}^{(d)}$ does not depend on $r$ and the pressure along the small dimensions trivially vanishes. It is then important to see if and how matter terms can alter this behaviour. For this purpose, we study the leading terms that have an explicit dependence on $r$ in the infinite sums appearing in 
eq. (54). We have numerically estimated that it is enough to consider the first $\mathrm{KK}$ and winding modes along a small direction, i.e. the terms with $\left\{N=\bar{N}=0, m_{i}=(1,0, \ldots, 0), n_{i}=0\right\}$ (as well as $m_{i}$ and $n_{i}$ exchanged) in eq. (54), plus the remaining $8-d$ inequivalent permutations. The energy $E_{\text {mat }}^{(d)}$ and pressures $P_{\text {mat }}^{(d)}$ and $P_{\text {mat }}^{(9-d)}$ along the large and small dimensions associated with these states are simply evaluated starting from the general expression (54). The equation of state for these leading order terms are:

$$
\begin{aligned}
& E_{\text {mat }}^{(d)}=-V_{d} C(\beta)^{(d)}\{ {\left[\frac{1}{r^{(d+1) / 2}} \frac{1-d}{2} K_{(d+1) / 2}\left(\frac{\beta}{r}\right)+\frac{1}{r^{(d+1) / 2}} \frac{\beta}{r} K_{(d+1) / 2}^{\prime}\left(\frac{\beta}{r}\right)\right]+} \\
& {\left.\left[r^{(d+1) / 2} \frac{1-d}{2} K_{(d+1) / 2}(\beta r)+r^{(d+1) / 2} \beta r K_{(d+1) / 2}^{\prime}(\beta r)\right]\right\}, } \\
& P_{\text {mat }}^{(d)}=V_{d} C(\beta)^{(d)}\left[\frac{1}{r^{(d+1) / 2}} K_{(d+1) / 2}\left(\frac{\beta}{r}\right)+r^{(d+1) / 2} K_{(d+1) / 2}(\beta r)\right], \\
& P_{\text {mat }}^{(9-d)=} V_{d} C(\beta)^{(d)} \beta\left[\frac{1}{r^{(d+3) / 2}} K_{(d-1) / 2}\left(\frac{\beta}{r}\right)-r^{(d+3) / 2} K_{(d-1) / 2}(\beta r)\right],
\end{aligned}
$$

where $K_{n}$ are modified Bessel functions, the prime denotes derivative with respect to $r$, and

$$
C(\beta)^{(d)}=\left(\frac{2 \pi}{\beta}\right)^{(d+1) / 2} \frac{(18-2 d)}{\pi} D(0)^{2} .
$$

Here $D(0)^{2}=256$ is a string degeneracy factor (see the Appendix). Note that we only consider the $p=1$ term in eq. (54), implying the approximation of the bosonic/fermionic statistics with the Maxwell-Boltzmann distribution. The pressure $P_{m a t}^{(d)}$ along the large dimensions is always positive, which aids expansion of the universe in addition to the pressure $P_{\text {rad }}^{(d)}$ from the massless states. The first and second terms in square brackets in eqs. (46) and (47) come from the KK and winding mode, respectively. The above equations (45)-(47) are all manifestly invariant under the duality symmetry (7) acting on the small dimensions, $r \rightarrow 1 / r$. Notice that the winding modes give rise to a standard positive pressure along the large dimensions (second term in (466)) but negative along the small ones (second term in (47)).

We numerically solved the dilaton-gravity equations (9)-(12), with $E=E_{\text {rad }}^{(d)}+E_{\text {mat }}^{(d)}, P^{(d)}=$ $P_{\text {rad }}^{(d)}+P_{\text {mat }}^{(d)}$ and $P^{(9-d)}=P_{\text {rad }}^{(9-d)}+P_{\text {mat }}^{(9-d)}$, by carefully taking into account the adiabaticity condition (30). The pressure $P_{\text {mat }}^{(9-d)}$ for the small dimensions vanishes at the self-dual critical radius $r=1$. Therefore it is expected that the effect of the massive states for the small dimensions is weak around $r \sim 1$. In fact we have numerically found that this is the case. As seen from the case (b) in fig. 4 the evolution of the small dimensions is hardly altered by including the massive mode for the initial value of $r$ very close to unity. From eq. (47) one notes that $P_{\text {mat }}^{(9-d)}<0$ for $0<r<1$ and $P_{\text {mat }}^{(9-d)}>0$ for $r>1$ (the asymptotic values are $P_{\text {mat }}^{(9-d)} \rightarrow 0$ for $r \rightarrow 0$ and $r \rightarrow \infty)$. This indicates that the pressure of the massive state makes the small dimensions contract for $0<r<1$ while its effect tends to expand the small dimensions for $r>1$.

The effect of the massive states emerges by choosing the initial values of $r_{0}$ that are slightly smaller or larger than unity. For $0<r_{0}<1$ with $\dot{\nu}_{0}>0$, the small dimensions can be larger than $r=1$ for large initial values of $\dot{\nu}$. In this case the small dimensions continue to grow after they cross $r=1$. When $\dot{\nu}_{0}$ is not large $\left(\dot{\nu}_{0} \ll 1\right)$, the massive effect can lead to the contraction 


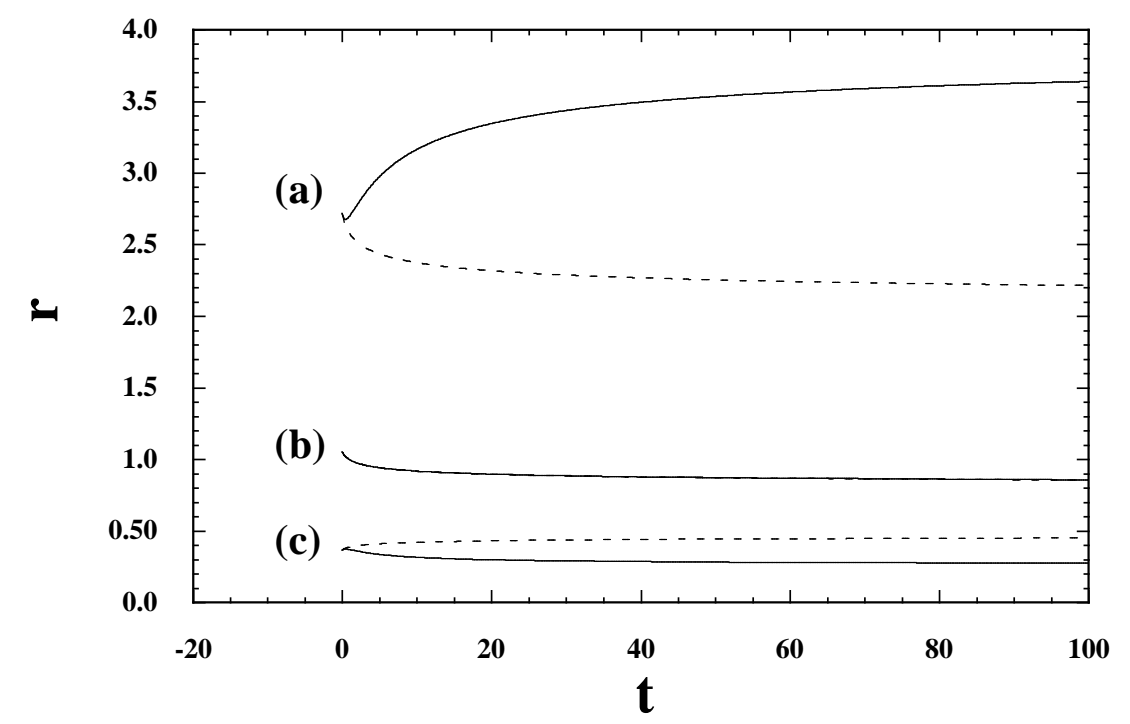

Figure 4: The evolution of the small dimensions for $d=4$ when the massive states are taken into account (solid curve). The dotted curves correspond to the case where the massive states are neglected (only massless states). We choose the initial conditions $\dot{\mu}_{0}=1.0, \mu_{0}=4.0, \psi_{0}=-16$, $\beta_{0}=12$ with (a) $\dot{\nu}_{0}=-0.1, \nu_{0}=1.0$, (b) $\dot{\nu}_{0}=-0.1, \nu_{0}=0.05$, (c) $\dot{\nu}_{0}=0.1, \nu_{0}=-1.0$.

of the small dimensions due to the negative pressure for $r<1$. As found from the case (c) in fig. 4. the small dimensions always increase in the massless case, whereas the small dimensions begin to contract if the massive effect is included. Therefore we can keep the $(9-d)$ dimensions small $(0<r<1)$ for these initial conditions.

We have also made numerical simulations for $r_{0}>1$. When $\dot{\mu}_{0}>0$ and $\dot{\nu}_{0}>0$, both large and small dimensions expand in the presence of positive pressures. If $\dot{\nu}_{0}$ is largely negative, the small dimensions contract by passing through $r=1$. Meanwhile, if $\left|\dot{\nu}_{0}\right| \ll 1$, the small dimensions can exhibit bouncing with $r>1$, instead of crossing $r=1$ [see the case (a) in fig. (4). This means that the radius $r$ can grow in the presence of the massive states. Since the small dimensions continue to expand after the bounce, this is not an ideal case where the small dimensions stay small. Nevertheless the small dimensions can be made small compared to the large dimensions as long as $\dot{\mu}_{0} \gtrsim \dot{\nu}_{0}$.

When the massive states are taken into account, this gives rise to an extra source term for the energy $E$ in eq. (91). Then $\left|\dot{\psi}_{0}\right|$ gets larger compared to the pure massless case. Typically this leads to the suppression of the r.h.s. of eq. (10) via the $e^{\psi}$ term, thereby yielding the smaller expansion rate $\dot{\mu}$ for the large dimensions. The large dimensions in the massive case grow slower relative to the massless case, as seen in fig. 5. In addition, the massive effect suppresses the growth of $\beta$, i.e. the temperature decreases faster in the massless case.

As expected, the massive terms get smaller as the initial value of $\beta$ is increased. For example, in the cases shown in fig. 4 and 5 the system is effectively described by the massless states for $\beta_{0}>15$. We also analyzed the behaviour of the system by varying the value $d$ with $1 \leq d \leq 8$. We found that the numerical results are quite similar to the case explained above $(d=4)$. As long as the conditions, $\dot{\mu}_{0} \gtrsim\left|\dot{\nu}_{0}\right|$ and $R_{0} \gtrsim r_{0} \sim 1$, are satisfied, the small dimensions are kept small, while the large ones expand as required in the presence of radiation and string matter. 


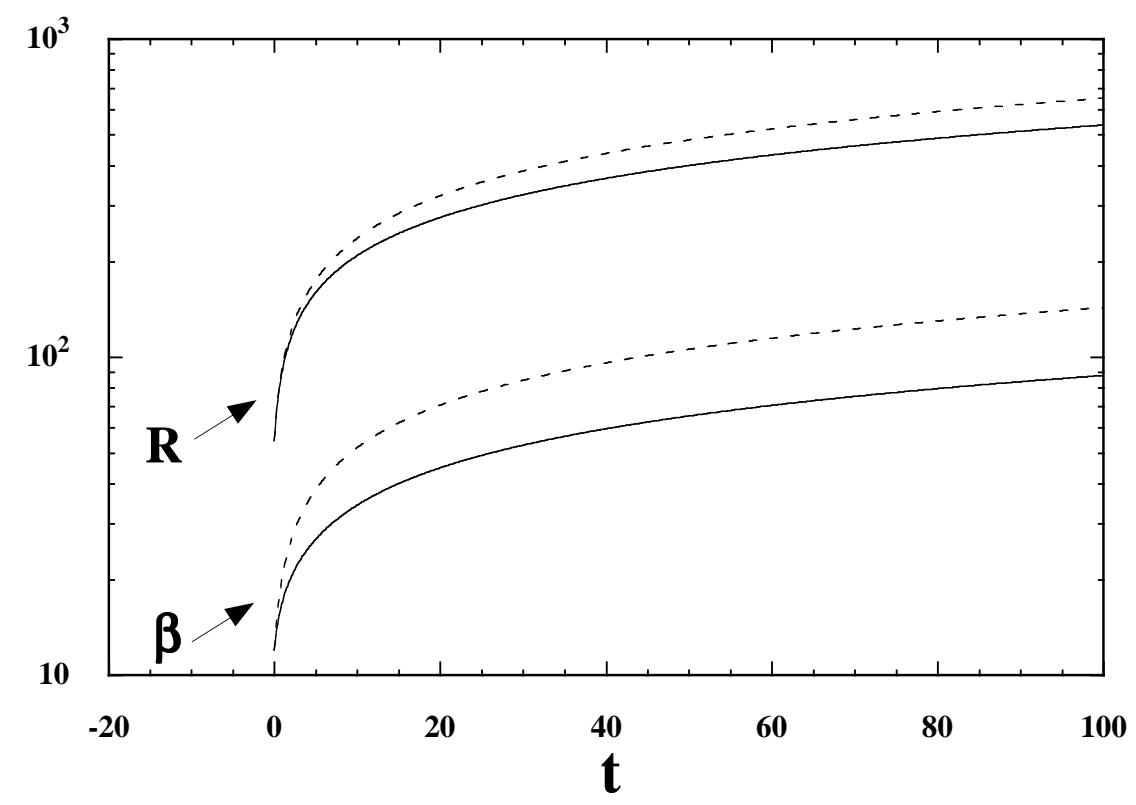

Figure 5: The evolution of the large dimensions and $\beta$ that corresponds to the case (c) in fig. 4. The dotted curves correspond to the case where the massive states are neglected (massless states only).

We have also considered the case with the conservation of KK and/or winding modes. Under this circumstance, the partition function must be taken summing only over the configurations that respect the conservation laws. As before, this is done by introducing a chemical potential corresponding to each conserved quantity in the partition function. It turns out that no significant changes are found compared to the case where no conservation laws are imposed.

\section{Conclusions}

We have studied string-gas cosmology at finite temperature in a toroidal universe. We make use of the dilation-gravity equations of motion, satisfying the $R \rightarrow 1 / R$ duality symmetry, to study the evolution of the system, which is assumed adiabatic. Our set up is as follows: the universe, initially homogeneous, isotropic and in thermal equilibrium, is filled with an ideal gas of closed strings. The 9 spatial dimensions, compactified on a 9-torus, evolve adiabatically starting from a Hagedorn regime.

In order to find an acceptable equation of state that describes such a system in string theory at finite temperature, we evaluate the energy and pressure in a microcanonical approach. Close to the Hagedorn regime, the scale factor $R$ exhibits a slow time evolution around $R \sim 1$, as shown in fig. 1. In this case the dynamics of the system is effectively described by a nearly constant energy and negligible pressure. We found that the analytic solution in the Hagedorn regime shows very good agreement with the full numerical result. No substantial changes are observed even when the conservation of Kaluza-Klein (KK) and winding modes is imposed, as long as the conserved charges are of order one.

We have also investigated a "low" temperature regime, in which the equation of state is 
derived in a canonical context. We first considered the dynamics of 3 "large" and 6 "small" compact dimensions in the presence of a pure gas of radiation (given by the massless states). It turns out, as expected, that there exist interesting cosmological solutions where the large dimensions continue to expand while the small dimensions remain nearly constant and small relative to the large ones (see fig. 2). The attractor solutions for the large dimensions can be described by the evolution of the standard radiation dominant phase in FRW cosmology whereas the small dimensions always asymptotically approach to a constant value. We also found bouncing solutions for the large dimensions if their Hubble rates are negative initially (see fig. [3).

We then analysed the case where the massive string states are taken into account in addition to the pure radiation. The presence of the massive states typically leads to a slower expansion of the "large" dimensions relative to the massless case (see fig. 51). Meanwhile the behaviour of the small dimensions strongly depends on the initial conditions for $r$ and $\dot{r}$, resulting in either expansion or contraction of the small dimensions (see fig. 4). The radius $r$ can be kept small as long as $r$ is initially close to unity, since the pressure vanishes at the duality symmetric radius $(r=1)$. The vanishing of the pressure at $r=1$ is a purely stringy effect, since it is due to winding modes, whose negative contribution compensates that of KK states. The important point is that, even in the presence of the massive state, there exist a wide range of the initial condition space for which the small dimensions are stabilized around the self-dual radius and are kept small relative to the large ones. These behaviours are found to be insensitive to the number of large dimensions, $d$. We also considered the case for the conservation of KK and winding modes and found no substantial change compared to the case without imposing the conservation laws.

In this work we did not investigate the later stage of the cosmological evolution. From the view point of cosmology, it is important to have an inflationary stage in order to solve the major cosmological problems (such as the flatness problem) as well as to generate the seeds for large-scale structure. One way to address this problem is to assume that the dilaton (or moduli) acquires mass, which may lead to inflation at later stages. Recently, for example, an interesting proposal was made by Parry and Steer [36], who showed that inflation can occur on a moving 3 -brane due to the nonminimal coupling of the dilaton to the brane matter. It was also shown in ref. [37] that brane inflation could result from the negative pressure of winding modes. Although it is not clear at present whether these proposals are directly applicable to our string-gas work, it is certainly of interest to extend the present analysis in that direction.

\section{Acknowledgements}

We thank Robert Brandenberger, Daisuke Ida, Ian Kogan, Yann Mambrini, and particularly Miguel Vazquez-Mozo, for very useful discussions and comments. M.B. thanks CONACyT (Mexico). The research of S.T. is financially supported from JSPS (No. 04942). B.B and S.T. are grateful to SISSA for their kind hospitality and support while much of the work of this project was completed.

This work was partially supported by the EC through the RTN network "The quantum structure of space-time and the geometric nature of fundamental interactions", contract HPRN-CT-200000131. 


\section{APPENDIX : The one-loop string partition function}

A thermal canonical partition function at temperature $T$ can be computed in the Euclidean approach by compactifying the time direction on a circle of length $\beta=1 / T$. We are then led to consider Type IIA/B string theory with all ten space-time directions compactified. For simplicity, we take the tori to be all rectangular (i.e. simple products of circles) and compute the free energy for the case in which $(9-d)$ spatial dimensions are compactified on small radii all equal to a common value denoted $r$, whereas the remaining $d$ directions are taken very large, and all equal to $R$. The free energy, after having unfolded the fundamental region of the world-sheet torus, takes the following form (see e.g. [38]):

$$
F^{(d)}(\beta)=-\frac{V_{d}}{2 \pi \sqrt{\alpha^{\prime}}} \int_{-\frac{1}{2}}^{\frac{1}{2}} d \tau_{1} \int_{0}^{\infty} \frac{d \tau_{2}}{\tau_{2}^{(3+d) / 2}}[\Lambda(r ; \tau)]^{9-d} \sum_{p=1}^{\infty} e^{-\frac{\beta^{2} p^{2}}{4 \pi \tau_{2}}}\left|M_{2}\right|^{2}(\tau),
$$

where

$$
\Lambda(r ; \tau)=\sum_{m, n=-\infty}^{+\infty} q^{\frac{1}{4}\left(\frac{m}{r}+n r\right)^{2}} \bar{q}^{\frac{\alpha^{\prime}}{4}\left(\frac{m}{r}-n r\right)^{2}},
$$

represents the contributions of the whole KK and winding modes along the small dimensions, $\tau$ is the so-called modular parameter of the world-sheet torus and $q=\exp (2 i \pi \tau)$. In deriving eq.(49) the winding modes along the large dimensions have been completely neglected and the sum over the KK modes has been approximated by an integral over continuous momenta. Note that $p$ in eq.(49) runs only over positive odd numbers and correspond to taking the correct quantum statistic for bosons and fermions. Taking only the term $p=1$ in the above sum corresponds to replacing the quantum bosonic/fermionic distribution with the classical Maxwell-Boltzmann distribution. $V_{d}$ is the volume of the large dimensions in $\left(4 \pi^{2}\right)$ units $^{6}$ :

$$
V_{d} \equiv \frac{1}{\left(4 \pi^{2}\right)^{d / 2}}(2 \pi R)^{d}=R^{d}
$$

The $M_{2}$ factor in eq. (49) encodes the contribution to the free energy of the whole tower of massive strings, and can be expanded in powers of $q$ :

$$
M_{2}(\tau)=\frac{\theta_{2}(\tau)^{4}}{\eta(\tau)^{12}}=\sum_{N=0}^{\infty} D(N) q^{N}
$$

Here $\theta_{2}$ and $\eta$ are modular functions on the torus (see e.g. 39 for an explicit expression) and $D(N)$ is the degeneracy factor at level $N(D(0)=16$, for example). The value of $N$ corresponds to each string mass level. The $\tau_{1}$ and $\tau_{2}$ integrals in eq. (49) can be easily performed. It is convenient to consider the term with $N=\bar{N}=m_{i}=n_{i}=0$ in eq. (49) separately from the remaining ones. This is the contribution of the purely massless states, which we will henceforth denote as $F_{\text {rad }}$ (where "rad" stands for radiation). We get

$$
F_{\text {rad }}^{(d)}=-\frac{R^{d}}{2 \pi} D(0)^{2} \Gamma\left(\frac{d+1}{2}\right)(4 \pi)^{(d+1) / 2} \zeta(d+1)\left(1-2^{-(d+1)}\right) \beta^{-d-1},
$$

\footnotetext{
${ }^{6}$ Recall that we are setting $\alpha^{\prime}=1$.
} 
where $\xi(x)$ is the Riemann zeta-function. The remaining "matter" terms give

$$
F_{m a t}^{(d)}=-\frac{V_{d}}{\pi} \sum_{m_{i}, n_{i}} \sum_{p=1}^{\infty} \sum_{N, \bar{N}}\left(\frac{2 \pi M}{\beta p}\right)^{(d+1) / 2} \delta_{m_{i} n_{i}+N-\bar{N}, 0} D(N) D(\bar{N}) K_{(d+1) / 2}(\beta p M),
$$

where $K_{(d+1) / 2}$ are modified Bessel functions, and

$$
M=M\left(m_{i}, n_{i}, r_{i}, N, \bar{N}\right) \equiv \sqrt{\sum_{i=1}^{9-d}\left(\frac{m_{i}^{2}}{r^{2}}+n_{i}^{2} r^{2}\right)+2(N+\bar{N})} .
$$

The total free energy is given by

$$
F^{(d)}=F_{\text {rad }}^{(d)}+F_{\text {mat }}^{(d)}
$$

The infinite sum over $N$ and $\bar{N}$ is not always convergent. In fact, the degeneracy factors $D(N)$, for large values of $N$, have a leading exponential behavior $D(N) \sim \exp (2 \pi \sqrt{2 N})$. On the other hand, for large values of its argument, the modified Bessel function $K_{n}(z)$ admits an asymptotic expansion whose leading term is $\sim \exp (-z)$. Hence, the sum over $N$ and $\bar{N}$ in eq. (54) converges only for $\beta>\beta_{H}=2 \pi \sqrt{2}$. The temperature $T_{H}=1 / \beta_{H}$ is the Hagedorn temperature.

As long as we deal with a range of energies where eq. (49) converges and no large energy fluctuations are present, we can work with the canonical ensemble. On the other hand, for high energy densities a microcanonical description has to be used. In this case the energy density of states is governed by the analytic structure of the canonical partition function $Z=\exp (-\beta F)$, in the complex $\beta$-plane. Taking into account the leading singularities of (49), we can parametrize the partition function as

$$
Z(\beta, R) \simeq \frac{e^{\Lambda(\beta, R)}}{\beta-\beta_{H}}\left(\frac{\eta_{K}}{\beta-\beta_{K}}\right)^{18}\left(\frac{\eta_{W}}{\beta-\beta_{W}}\right)^{18},
$$

where $\eta_{K}$ and $\eta_{W}$ are defined by eq. (16) with $\beta_{K}=\beta_{H}-\eta_{K}$ and $\beta_{W}=\beta_{H}-\eta_{W} . \Lambda(\beta, R)$ is an entire function in $\beta$. The microcanonical energy distribution function $\Omega(E)$, is then given by

$$
\Omega(E)=\int_{\beta_{H}-i \infty}^{\beta_{H}+i \infty} \frac{d \beta}{2 \pi i} Z(\beta, R) e^{\beta E} \simeq \sum_{i=H, K, W} \oint_{C_{i}} \frac{d \beta}{2 \pi i} Z(\beta, R) e^{\beta E},
$$

where $C_{H}, C_{K}$ and $C_{W}$ are the three contours encircling respectively the poles in $\beta_{H}, \beta_{K}$ and $\beta_{W}$ in the complex $\beta$-plane. The entropy and the rest of the thermodynamical quantities easily follow from eq. (58).

\section{References}

[1] I. Antoniadis, Phys. Lett. B 246 (1990) 377;

I. Antoniadis, C. Munoz and M. Quiros, Nucl. Phys. B 397 (1993) 515 arXiv:hep-ph/9211309.

[2] R. Brandenberger and P. M. Ho, Phys. Rev. D 66 (2002) 023517 [AAPPS Bull. 12N1 (2002) 10] arXiv:hep-th/0203119;

S. Alexander, R. Brandenberger and J. Magueijo, arXiv:hep-th/0108190,

S. Alexander and J. Magueijo, arXiv:hep-th/0104093. 
[3] J. Maldacena, arXiv:astro-ph/0210603.

[4] G. Veneziano, Phys. Lett. B 265, 287 (1991);

K. A. Meissner and G. Veneziano, Phys. Lett. B 267, 33 (1991).

[5] M. Gasperini and G. Veneziano, Astropart. Phys. 1 (1993) 317 arXiv:hep-th/9211021.

[6] J. E. Lidsey, D. Wands and E. J. Copeland, Phys. Rept. 337, 343 (2000) arXiv:hep-th/9909061.

[7] M. Gasperini and G. Veneziano, arXiv:hep-th/0207130

[8] J. Khoury, B. A. Ovrut, P. J. Steinhardt and N. Turok, Phys. Rev. D 64, 123522 (2001) arXiv:hep-th/0103239; Phys. Rev. D 66, 046005 (2002) arXiv:hep-th/0109050.

[9] P. J. Steinhardt and N. Turok, arXiv:hep-th/0111030 Phys. Rev. D 65, 126003 (2002) arXiv:hep-th/0111098.

[10] H. Liu, G. Moore and N. Seiberg, JHEP 0210, 031 (2002) arXiv:hep-th/0206182;

S. Elitzur, A. Giveon, D. Kutasov and E. Rabinovici, JHEP 0206, 017 (2002) arXiv:hep-th/0204189.

[11] R. H. Brandenberger and C. Vafa, Nucl. Phys. B 316 (1989) 391.

[12] A. A. Tseytlin and C. Vafa, Nucl. Phys. B 372 (1992) 443 arXiv:hep-th/9109048.

[13] A. A. Tseytlin, Class. Quant. Grav. 9 (1992) 979 arXiv:hep-th/9112004.

[14] M. A. Osorio and M. A. Vazquez-Mozo, Mod. Phys. Lett. A 8 (1993) 3111 arXiv:hep-th/9305137; Mod. Phys. Lett. A 8 (1993) 3215 arXiv:hep-th/9305138.

[15] G. B. Cleaver and P. J. Rosenthal, Nucl. Phys. B 457 (1995) 621 arXiv:hep-th/9402088.

[16] M. Sakellariadou, Nucl. Phys. B 468 (1996) 319 arXiv:hep-th/9511075.

[17] S. Alexander, R. H. Brandenberger and D. Easson, Phys. Rev. D 62 (2000) 103509 arXiv:hep-th/0005212.

[18] R. Brandenberger, D. A. Easson and D. Kimberly, Nucl. Phys. B 623 (2002) 421 arXiv:hep-th/0109165.

[19] D. A. Easson, arXiv:hep-th/0110225.

[20] R. Easther, B. R. Greene and M. G. Jackson, Phys. Rev. D 66 (2002) 023502 arXiv:hep-th/0204099.

[21] S. Watson and R. H. Brandenberger, arXiv:hep-th/0207168

[22] R. Easther, B. R. Greene, M. G. Jackson and D. Kabat, arXiv:hep-th/0211124

[23] Y. Leblanc, Phys. Rev. D 38 (1988) 3087.

[24] T. Damour and A. M. Polyakov, Gen. Rel. Grav. 26, 1171 (1994) arXiv:gr-qc/9411069. 
[25] T. Damour, F. Piazza and G. Veneziano, Phys. Rev. D 66, 046007 (2002) arXiv:hep-th/0205111; Phys. Rev. Lett. 89, 081601 (2002) arXiv:gr-qc/0204094.

[26] S. Randjbar-Daemi, A. Salam and J. Strathdee, Phys. Lett. B 135 (1984) 388.

[27] L. Alvarez-Gaume and M. A. Vazquez-Mozo, arXiv:hep-th/9212006, section 4.3 and references therein.

[28] R. Hagedorn, Suppl. Nuovo Cimento 3 (1965) 147.

[29] N. Deo, S. Jain and C. I. Tan, Phys. Lett. B 220 (1989) 125.

[30] N. Deo, S. Jain and C. I. Tan, Phys. Rev. D 40 (1989) 2626.

[31] S. W. Hawking, Phys. Rev. D 13 (1976) 191.

[32] M. Axenides, S. D. Ellis and C. Kounnas, Phys. Rev. D 37 (1988) 2964;

M. J. Bowick and L. C. Wijewardhana, Phys. Rev. Lett. 54 (1985) 2485.

[33] J. J. Atick and E. Witten, Nucl. Phys. B 310 (1988) 291.

[34] M. Gasperini, M. Maggiore and G. Veneziano, Nucl. Phys. B 494, 315 (1997) arXiv:hep-th/9611039;

R. Brustein and R. Madden, Phys. Rev. D 57, 712 (1998) arXiv:hep-th/9708046.

[35] S. Tsujikawa, R. Brandenberger and F. Finelli, Phys. Rev. D 66, 083513 (2002) arXiv:hep-th/0207228.

[36] M. F. Parry and D. A. Steer, JHEP 0202, 032 (2002) arXiv:hep-ph/0109207.

[37] S. Abel, K. Freese and I. I. Kogan, JHEP 0101, 039 (2001) [archiv:hep-th/0005028; arXiv:hep-th/0205317.

[38] E. Alvarez and M. A. Osorio, Phys. Rev. D 36, 1175 (1987);

K. H. O'Brien and C. I. Tan, Phys. Rev. D 36 (1987) 1184.

[39] J. Polchinski, String Theory, Volume 1, Cambridge, page. 214-216. 